\title{
COMPRIMENTO TOTAL DO INTESTINO EM BOVINOS AZEBUADOS
}

\author{
Orlando Marques de PAIVA *
}

Vicente BORELLI **

\section{RFMV-A/20}

PAIVA, O.M. \& BORELLI, V. Comprimento total do intestino em bovinos azebuados. Rev. Fac. Med. vet. Zootec. Univ. S.PaulQ, 14(1):171-178, 1977

RESUMO: Foi estudado o comprimento dos vários tratos do intestino bem como seu compri mento total, em 40 bovinos azebuados, 20 machos e 20 fêmeas, adultos e encontraram as seguintes medidas:

$$
\begin{aligned}
& \text { intestino delgado ...................115 m } \pm 2,73 \\
& \text { ceco } \ldots . . . . . . . .0,51 \mathrm{~m} \pm 0,11 \\
& \text { colon } \text { reto . ...................6,80 m } \pm 0,80 \\
& \text { intestino grosso ..................7,31 } \mathrm{m} \pm 0,75 \\
& \text { comprimento total ................38,84 } m \pm 3,26
\end{aligned}
$$

A análise estatistica näo mostrou diferenças significantes entre os sexos, nem correlaçuo entre o comprimento total do intestino como peso ou o comprimento da carcaça.

UNITERMOS: Intestinos, comprimento *; Bovinos azebuados *.

\section{INTRODUÇÃO E LITERATURA}

A anatomia dos zebuínos demanda ainda bastante labor de quantos se votam às pesquisas morfológicas macro e microscó picas pois, a todo momento surpreendemos dificuldades em acomodar os fatos por ela proporcionados com as indicações dos tratadistas, cujos dados se fundamentam, provavelmente, nos bovinos europeus.

As informaçôes obtidas em trabalhos anteriores, sobre a distância do torus pyloricus à papilla duodeni hepatica (PAIVA \& ASSIS RIBEIRO ${ }^{20}$ - 1948) e desta à papilla duodeni pancreática (PAIVA \& D'ERRI$\left.\mathrm{CO}^{21}-1949\right)$ demonstrando a existência de diferenças significantes, quando se comparam os resultados obtidos em bovinos azebuados, com valores fornecidos pelos compên. dios de Veterinária; ainda a hipótese de que esta diferenca deveria imputar-se ao menor comprimento do intestino dos bovinos mestiços de zebu e a grande importância do conhecimento das dimensões do trato intestinal desses animais, que representam o maior contingente de bovinos do país, constituiram os principais motivos da pesquisa realizada.

Dados relativos ao comprimento de segmentos intestinals dos bovinos, encontrados em tratados reservados à Anatomia Veterinária, procuramos condensar em tabela ora apresentada:

\footnotetext{
* Professor Catedrático.

* Professor Titular.

Departamento de Cirurgia e Obstetrícia da Faculdade de Medicina Veterinária e Zootecnia da USP.
} 


\begin{tabular}{|c|c|c|c|c|c|}
\hline AUTORES & ANO & $\begin{array}{l}\text { INTEST. } \\
\text { DELGADO }\end{array}$ & $\begin{array}{l}\text { INTEST, } \\
\text { GROSSO }\end{array}$ & CECO & TOTAL \\
\hline CARADONA \& BOSSI & s.d. & $40-50$ & - & 0,80 & - \\
\hline MONGIARDINO ${ }^{18}$ & 1905 & - & - & 0,80 & - \\
\hline MONTANE'\& BOURDELLE 19 & 1917 & 40 & $7,70-8,75$ & $0,70-0,75$ & $\pm 47,70-48,75$ \\
\hline LEPOUTRE $E^{14}$ & 1921 & 40 & - & - & - \\
\hline$L E S B R E^{15}$ & 1922 & 40 & $8-10$ & - & - \\
\hline$C A R A D O N A^{3}$ & 1930 & $40-45$ & - & 0,80 & - \\
\hline FAVILLI ${ }^{8}$ & 1931 & 40 & $7,70-8,80$ & $0,70-0,80$ & $\pm 47,70-48,80$ \\
\hline ELLENBERGER \& BAUM ${ }^{7}$ & 1932 & $40-49$ & $6,40-11$ & - & $39-63$ \\
\hline MARTIN \& SCHAUDER ${ }^{16}$ & 1935 & $30-45$ & $6,40-10$ & - & $39-59$ \\
\hline$S C H M A L T Z^{22}$ & 1958 & - & 8,20 & - & - \\
\hline$F A V I L L I^{19}$ & 1943 & 40 & $7-8$ & - & - \\
\hline$B R U N I$ \& ZIMMERL $L^{2}$ & 1949 & $40-45$ & $8-10$ & - & \\
\hline ZIMMERL ${ }^{26}$ & 1949 & 40 & $\pm 8,10-9,1 \theta$ & 0,50 & $\pm 48,10-49,10$ \\
\hline$K L I M O V^{13}$ & 1953 & - & $6,40-10$ & - & - \\
\hline DOBBERSTEIN \& KOCH ${ }^{5}$ & 1954 & - & - & - & $39-63$ \\
\hline$M A S S U I^{17}$ & 1960 & $40-49$ & $6,40-11$ & $0,50-0,60$ & $39-63$ \\
\hline NICKEL \& SCHUMMER ${ }^{23}$ & 1960 & $27-49$ & $6,50-14$ & $0,50-0,70$ & $33-63$ \\
\hline $\begin{array}{l}\text { GONZALESY GARCIA \& } \\
\text { ALVAREZ }\end{array}$ & 1961 & 45 & $10-12$ & - & $55-57$ \\
\hline$S C H W A R Z E$ \& SCHRODER ${ }^{24}$ & 1962 & $27-49$ & $6,50-14$ & - & $33,50-63$ \\
\hline KATO ${ }^{12}$ & 1963 & \pm 41 & \pm 10 & 0,75 & - \\
\hline SISSON \& GROSSMAN ${ }^{25}$ & 1965 & 40 & $\pm 11,05$ & 0,75 & $\pm 5 l, 05$ \\
\hline
\end{tabular}

BLACK, SEMPLE \& LUSH $^{1}$ (1934), por sua vez, ao comparar o comprimento do intestino de mestiços meio sangue, Zebu Hereford e Zebu Shorthorn, com bovinos puro sangue Hereford e Shorthorn, todos animais jovens, não registram diferenças consideradas estatisticamente significantes.

Já, de GIROLAMO ${ }^{10}$ (1950), estudando comparativamente a morfologia e o comprimento intestinal, entre Bos bubalus e Bos taurus, estes 3 machos e 7 fêmeas com idade variando de 1 a 7 anos, informa que o comprimento total do intestino dos taurinos compreende, na maior parte das vezes, valores que oscilam de 47 a 50 metros. Considerando os bovinos, o $\mathrm{A}$. fornece ainda os dados relativos a cada animal, o que nos permite conhecer as dimensões limites dos segmentos, bem como as médias, que calculadas apontam: para o intestino delgado 39,42 $\mathrm{m}$, sendo o valor mínimo encontrado 35,50 m e o máximo $42,00 \mathrm{~m}$; para o ceco $0,70 \mathrm{~m}$, medindo de $0,50 \mathrm{~m}$ a $0,80 \mathrm{~m}$; para o colon $\mathrm{e}$ reto $7,30 \mathrm{~m}$, variando de $6,03 \mathrm{~m}$ a $7,95 \mathrm{~m}$ e finalmente para o intestino total, a média de $47,52 \mathrm{~m}$, atingindo os valores limites de $42,03 \mathrm{~m}$ a $50,70 \mathrm{~m}$. Verificou também o A., correlação positiva ao confrontar as dimensões intestinais com a altura e comprimento dos animais.

\section{MATERIAL E MÉTODO}

Realizamos mensuraçбes do trato digestório, tomadas do toro pilórico ao ânus, em 40 bovinos azebuados, 20 machos e 20 fêmeas, que exibiam dentiçăo definitiva completa, ou seja, com 49,9 meses no mínimo (tabela de CHIEFFI, PAIVA e VEI$\mathrm{GA}^{5}$ - 1948) procedentes de diversas zonas de criação e abatidos no frigorifico Armour de São Paulo.

Logo após o sacrifício, retirávamos a peça em bloco, já libertada do fígado, incisando transversalmente o abomaso e a seguir, seccionávamos o mesentério ao longo da pequena curvatura, eliminando também o 
epiplo e o pâncreas. Convenientemente retificado e evitando estirá-lo, colocávamos o material sobre plano horizontal, registrando então sempre dentro de período não superior a $\mathbf{5}$ horas após o sacrifício, as medidas dos diferentes segmentos, anotando-as em ficha, da qual constavam, além dos enumerados, informes relativo ao peso das meias carcaças e à distância entre as junturas das sétima e oitava vértebras torácicas e lombo sacra.
No estudo estatístico dos dados, utilizamos o teste de hipóteses de Student (testt) e a análise de correlação.

\section{RESULTADOS}

No material por nós examinados, os comprimentos dos diversos segmentos intestinais, alcançaram, em metros, as médias glo. bais e respectivos desvios padrões, que reunimos em tabela apresentada a seguir:

\begin{tabular}{l|c|c|c|c|c}
\hline \multirow{2}{*}{$\begin{array}{c}\text { Segmentos } \\
\text { intesti- }\end{array}$} & \multirow{2}{*}{$\begin{array}{c}\text { INTESTINO } \\
\text { DELGADO }\end{array}$} & \multicolumn{3}{|c|}{ INTESTINO GROSSO } & \multirow{2}{*}{$\begin{array}{c}\text { COMPRIMENTO } \\
\text { TOTAL }\end{array}$} \\
\cline { 3 - 5 } Mais & CECO & COLON+RETO & TOTAL & TOT \\
\hline MACHOS & $30,72 \pm 1,99$ & $0,51 \pm 0,12$ & $6,56 \pm 0,95$ & $7,07 \pm 1,04$ & $37,78 \pm 2,73$ \\
& & & & \\
FEMEAS & $31,58 \pm 3,24$ & $0,52 \pm 0,10$ & $7,03 \pm 0,50$ & $7,55 \pm 0,56$ & $39,13 \pm 3,64$ \\
TOTAL & $31,15 \pm 2,73$ & $0,51 \pm 0,11$ & $6,80 \pm 0,80$ & $7,31 \pm 0,75$ & $38,46 \pm 3,26$ \\
\hline
\end{tabular}

Como valores limites, respectivamente máximo e mínimo, registramos para o intestino delgado $40,07 \mathrm{~m}$ e $27,14 \mathrm{~m}$; ceco $0,94 \mathrm{~m}$ e $0,35 \mathrm{~m}$, cólon e reto $9,67 \mathrm{~m}$ e $4,95 \mathrm{~m}$, intestino grosso $10,61 \mathrm{~m}$ e $5,44 \mathrm{~m}$ e finalmen. te para o comprimento total, $48,03 \mathrm{~m}$ e $33,62 \mathrm{~m}$.

Os achados individuais relativos ao peso das carcaças, à distância entre as junturas da sétima e oitava vértebras torácicas e lombo sacra, bem como o comprimento dos diversos segmentos intestinais de cada animal, encontram-se nos quadros 1 (machos) e 2 (fêmeas).

As diferenças obtidas, relativamente aos sexos, quando analisadas estatisticamente (test t) mostraram-se em todos os casos, destituidas de significância, ao nível de rejeição adotado de $5 \%$.

Por outro lado, năo foi evidenciada correlação ao se confrontar o comprimento total do intestino com o peso das carcaças ou com a medida da distância entre o disco intervertebral correspondente à juntura das sétima e oitava vértebras torácicas e a articulação lombo sagrada.

\section{COMENTÅRIOS}

Embora os dados fornecidos pelos tratadistas (tabela 1) não possam ser confrontados estatisticamente com os resultados obtidos na presente pesquisa, por nảo apresentarem os AA. informações relativas à raça, idade, sexo, bem como o número de animais, provavelmente de origem européia, que estudados serviram de base para tais citações, podemos verificar que as médias correspondentes ao comprimento do intestino delgado $(31,15 \mathrm{~m})$, do intestino grosso $(7,31 \mathrm{~m})$ do ceco $(0,51 \mathrm{~m})$ e do intestino total $(38,84 \mathrm{~m})$, por nós encontradas são na maioria das vêzes inferior ou em alguns casos pouco superior ao limite mínimo registrado nos compêndios de Anatomia Veterinária.

Esta comparação, por outro lade, parece confirmar a hipótese de PAIVA \& ASSIS RIBEIRO $^{20}$ e de PAIVA \& D'ERRICO ${ }^{21}$, que admitem ter 08 zebuínos, as distâncias do torus pyloricus a papilla duodeni major $\mathrm{e}$ desta à papilla duodeni minor dimensões menores, quando confrontadas com as do Bos taurus, face ao maior comprimento intestinal 


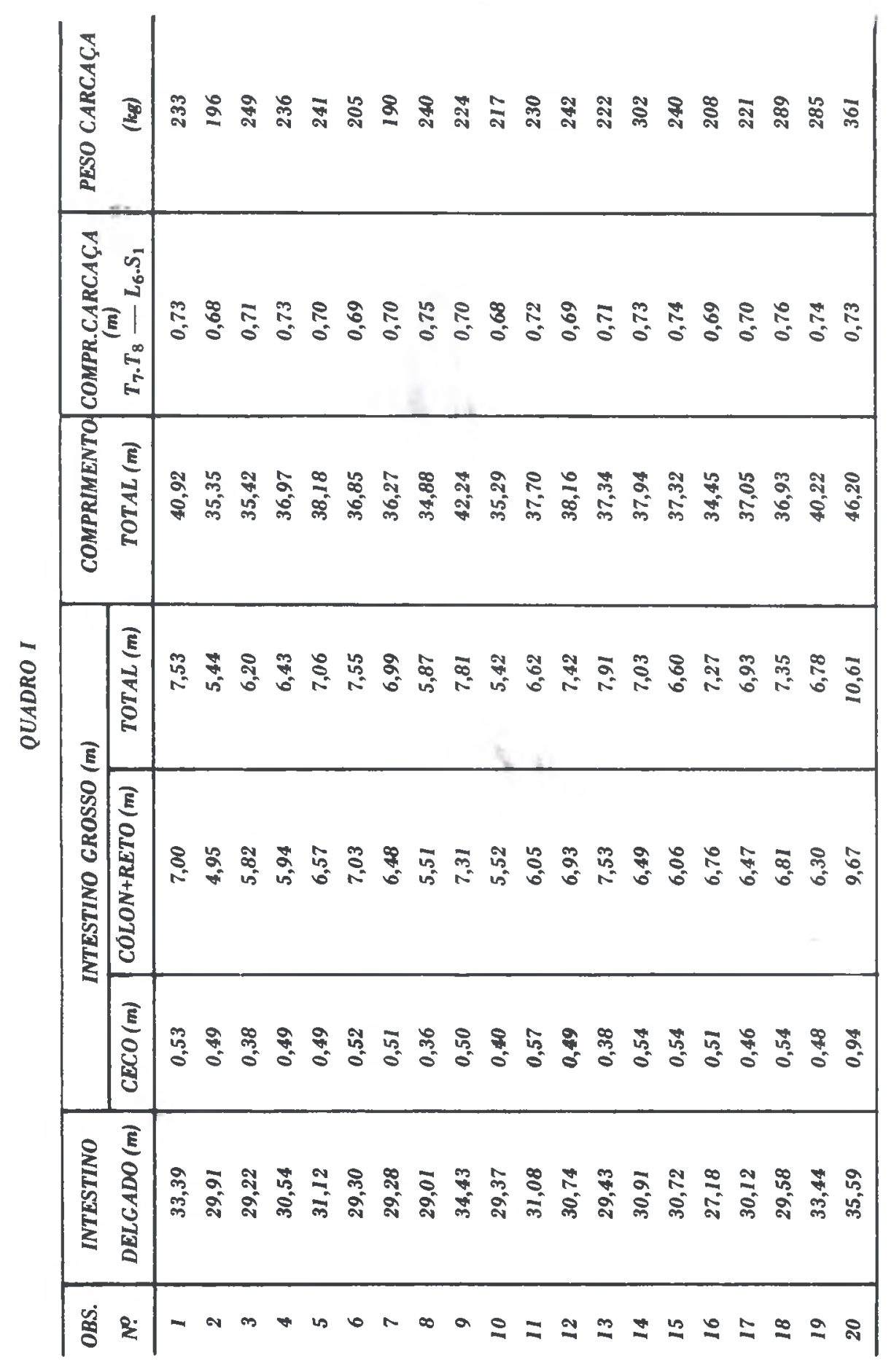




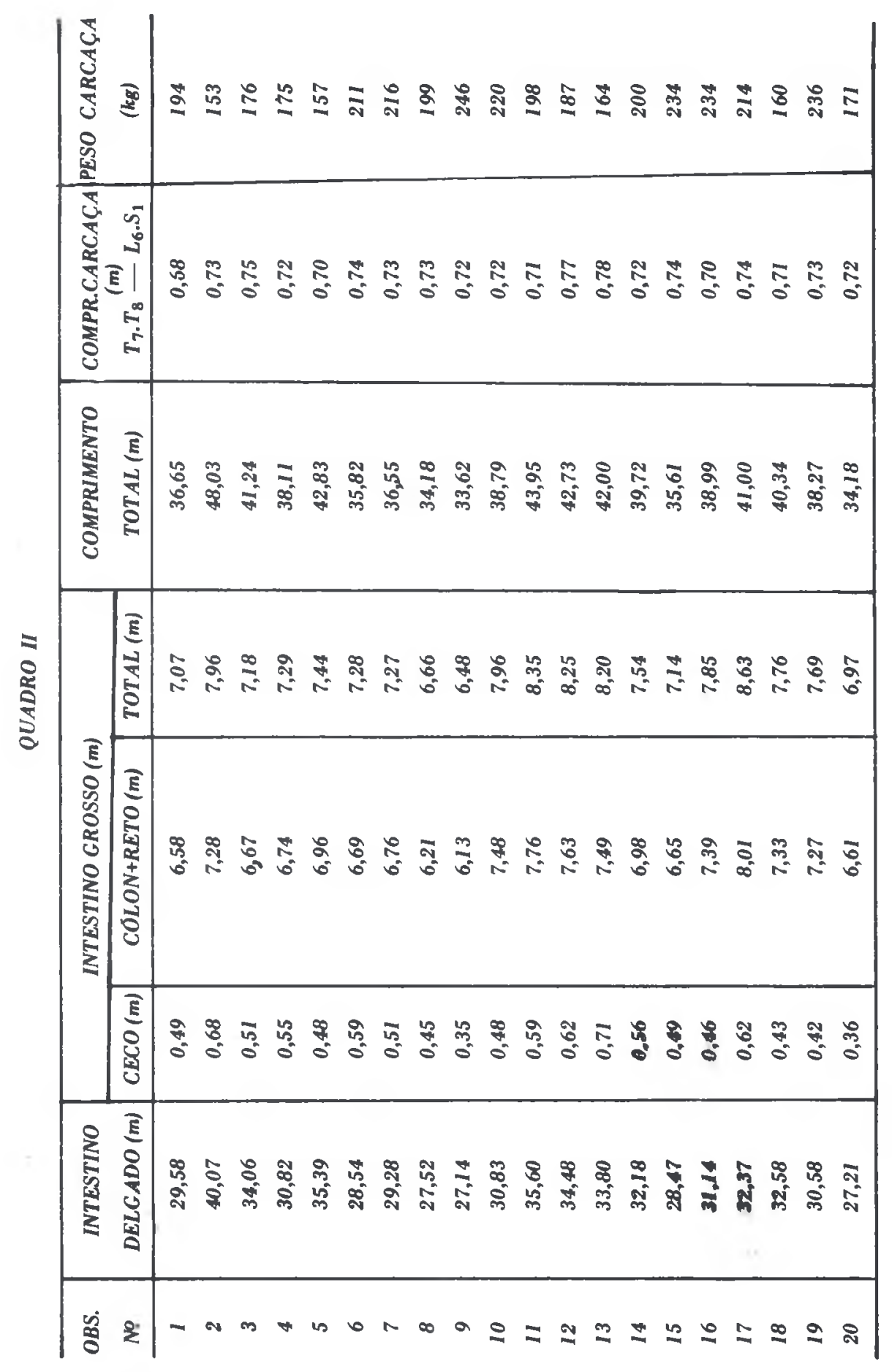

Rev. Fac. Med, vet. Zootec. Univ. S. Paulo, 14(1):171-178, 1977. 
destes animais, muito embora BLACK, SEMPLE \& LUSH $^{1}$ ao medir o intestino de mestiços meio sangue Zebu Hereford e Zebu Shorthorn, e de bovinos puro sangue Hereford $\mathrm{e}$ Shorthorn nấo tenham assinalado, provavelmente por trabalharem com animais jovens, diferenças consideradas estatistica* mente significantes.

Já, no estudo comparativo realizado por de GIROLAMO ${ }^{10}$, relativo ao comprimento dos intestinos de Bos bubalus e de Bos taurus, observamos que o A. registra para estes valores cujas médias referentes ao intestino delgado $(39,42 \mathrm{~m})$, colon e reto $(7,30 \mathrm{~m})$, ceco $(0,70 \mathrm{~m})$ e intestino total $(47,52 \mathrm{~m})$ mostram-se visivelmente superiores às por nós assinaladas para bovinos azebuados, não sendo todavia possível, também neste caso, o confronto estatístico, uma vez que aquelas medidas foram realizadas em animais com idade cronológica diversa, isto é, variando de 1 a 7 anos.

Por fim, analisando estatisticamente, os resutlados ora obtidos, verificamos que as diferenças existentes, ao nivel de $5 \%$ independem do sexo, bem como năo se evidencia correlação entre o comprimento total do intestino com o peso da carcaça ou com o seu comprimento, tomado entre as junturas das sétima e oitava vértebras torácicas e lombo sacra.

\section{CONCLUSÕES}

As medidas obtidas em 40 tratos intestinais de bovinos azebuados, 20 machos e 20 fêmeas, com no mínimo 49,9 meses de idade, permite.nos as seguintes conclusões:

1 - o intestino delgado, em média, apresenta o comprimento de $30,72 \mathrm{~m} \pm 1,99$ para os machos e $31,58 \mathrm{~m} \pm 3,24$ para as fêmeas, a média geral $31,15 \mathrm{~m} \pm 2,73$ e como valores máximo e mínimo, respectivamente, 40,07 m e 27, 14 m;

2 - o ceco, em média, mostra-se com $0,51 \mathrm{~m} \pm 0,12$ nos machos $\mathrm{e}$ $052 \mathrm{~m} \pm 0,10$ nas fêmeas tendo por média geral $0,51 \mathrm{~m} \pm 0,11$ e por limites $0,94 \mathrm{~m}$ e $0,35 \mathrm{~m}$;

3 - o cólon e reto, em média, exibe 6,56 $\mathrm{m} \pm 0,95$ nos machos e $7,03 \mathrm{~m} \pm 0,50$ nas fêmeas, obtendo como média geral $6,80 \mathrm{~m} \pm 0,80$ e como valores extremos $9,67 \mathrm{~m}$ e $4,95 \mathrm{~m}$;

4 - o intestino grosso, em média, alcança $7,07 \mathrm{~m} \pm 1,04$ nos machos e 7,55 $\mathrm{m} \pm 0,56$ nas fêmeas, sendo a média geral $7,31 \mathrm{~m} \pm 0,75$ e os valores limites $10,61 \mathrm{~m} \mathrm{e} 5,44 \mathrm{~m}$;

5 - o comprimento total do intestino desses ruminantes, em média, atinge 37,78 $\mathrm{m} \pm 2,73$ nos machos e $39,13 \mathrm{~m} \pm 3,64$ nas fêmeas, apresentando como média geral $38,46 \mathrm{~m} \pm 3,26$ e como valores máximo e mínimo, sucessivamente, 48,03 e $33,62 \mathrm{~m}$;

6 - As diferenças encontradas relativamente ao sexo,foram atribuídas ao acaso, uma vez que a análise estatística dos dados, jamais revelou ocorrencia de significância, ao nível de 5\%;

7 - confrontando o comprimento total do intestino com o peso das carcaças ou com a medida da distância entre as junturas das sétima e oitava vertebras torácicas e a articulação lombo sagrada, não foi demonstrada correlação. 
PAIVA, O.M. \& BORELLI, V. The lenght of the intestine in Zebu cattle. Rev. Fac. Med. vet. Zootec. Univ. S.Paulo, 14(1):171-178, 1977.

SUMMARY: The A.A. studied the length of the varions enteric tracts as well as their full length in 40 adult zebu cattle (20 males and 20 females) and achieved the following overage, once there was no significant difference concerning sex:

$$
\begin{aligned}
& \text { thin intestine ......................... } \\
& \text { caecun ........................... } 0.51 \pm 0.11 \\
& \text { colon and rectun } \ldots \ldots \ldots \ldots \ldots \ldots \ldots \ldots \ldots \ldots \ldots, 6.80 \pm 0.80 \\
& \text { gross intestine ......................... } 71 \pm 0.75 \\
& \text { full length ........................38.84 } \pm 3.26
\end{aligned}
$$

On the other hand, there was no correlation between the full length of the intestine and the weight or lenght of the carcasses.

UNITERMS: Intestines, lenght *; Zebu cattle *

\section{REFERẼNCIAS BIBLIOGRĀFICAS}

1 - BLACK, W.H.; SEMPLE, A.T.; LUSH, J.L. Beef production and quality as influence by croosing Brahman with Hereford and Shorthorn cattle. Tech, Bull. U.S. Dep, Agric, (417) 1934.

2 - BRUNI. A.C. \& ZIMMERL. U. Anatomia degli animali domestici. Milano, Francesco Vallardi, 1949, v.2.

3 - CARADONNA, G.B. In: ZIMMERL U. Trattado di anatomia veterinária. Milano, Francesco Vallardi, $1930, v 2$.

4 - CARADONNA, G.B. In: BOSSI, V.; CARADONNA, G.B.; SPAMPANI, G.; VARALDI, L.; ZIMMERL, U. Trattado di anatomia veterinaria. Milano Francesco Vallardi, s.d., v.2.

5 - CHIEFFI, A.; PAIVA, O.M.; VEIGA, J.S. Contribuiç̃̃o para o estudo da cronologia dentária do zebu. Rev. Fac. Med, vet., São Paulo, 3: 251-69, 1948.

6 - DOBBERSTEIN, J. \& KOCH, T. In: PIEKOS, M.; PILARSKI, W.; ROSKOSZ, T. Obserwacje nad dtugoscia jelita u zubra Bison bonasus L. Folia morph., Warszawa, 9: 69-79, 1958.

7 - ELLENBERGER, W. \& BAUM, H. Hand buch der vergleichenden Aratomie det Haustiere. 17 auf. Berlin, Julius Springer, 1932.

8 - FAVILLI, N. Nozioni comparate di anatomia e fisiologia degli animali rura. li. Torino, Unione Tipografico, Editrice Torinesse, 1931.
9 - FAVILLI, N. Nozione comparate di anatomia e fisiologia degli animali agricoli. Milano, Francesco Vallardi, 1943.

10 - GIROLAMí, A. Rilievi campanativi sulla morfologia sulla lunghezza dell'intestino fra Bos taurus e Bos bubalus. Nuova Vet., 1:16, 1950 .

11 - GONZALEZ Y GARCIA, J. \& ALVAREZ, R.G. Anatomia comparada de los animales domesticos. 7. ed Madrid, Gráficas Canales, 1961.

12 - KATO, K. Katiku hikaku Kaibon zusitsu. (Esposição gráfica da anatomia comparada dos animais domésticos). 6.ed. Tokio, Yokendo, 1963. v.1.

13 - KLIMOV, A. In: DOBBERSTEIN, J.; KOCH, T. apud PIEKOS, M.; PILARSKI, W.; ROSKOSZ, T. Obserwacje nad dtugoscia jelita u zubra Bison bonasus L. Folia Morph, Warszawa, 9: 69-79, 1958 .

14 - LEPOUTRE, L. Notes du cours d'anato. mie comparée des animaux domestiques. Gembloux J. Duculot, 1921.

15 - LESBRE, F.X. Précis d'anatomie comprarée des animaux domestiques. Raris, J. B. Baillière, 1922, v.1.

16 - MARTIN, P. \& SCHAUDER, W. Lehrbuch der Anatomie der Hanstiere. 3, Auf. Stuttgart, Schickhardt \& Ebner, 1935, v. 3 .

17 - MASSUI, K. Katiku hikaku kaibon ku., /Anatomia comparada dos anlmais domésticos./ 7. ed. Tokiq Yokendo, 1960. 
18 - MONGIARDINO, T. In: GIROLAMO, A. Rilieve comparativi sulla mórfo. logia e sulla lunghezza dell'intestino fra Bos taurus e Bos bubalus. Nuova vet., $1: 1-6,1950$.

19 - MONTANE, L. \& BOURDELLE, E. Anatomie régionale des animaux domestiques. Paris, J.B. Baillière, 1917.v.2.

20 - PAIVA, O.M. \& ASSIS RIBEIRO,P. Distâncis do Torus pyloricus à Papilla du. odeni hepatica em bovinos mestiços de zebu. Rev. Fac. Med. vet., São Paulo, 3: 223.46, 1948.

21 - PAIVA, O.M. \& D'ERRICO, A.A. Distância da Papilla duodeni hepotica à Pa pilla duodeni pancreatica em bovinos mestiços de zebu. Rev. Fac. Med, vet., São Paulo, 4: 5-26, 1949.

22 - SCHMALTZ, R. In: DOBBERSTEIN, J.; KOCH, T. apud PIEKOS, M.; PILARSKI, W.; ROSKOSZ, R. Obser- wacje nad dtugoscia jelita u zubra Bison bonasus L. Folia morph, Warszawa, 9: 69-79, 1958.

23 - SCHUMMER, A. \& NICKEL, R. In: NICKEL, R.; SCHUMMER, A.; SEIFERLE, E. Lehrbuch der Anatomie der Haustiere. Berlin, Paul Parey, 1960, v.2.

24 - SCHWARZE, E. \& SCHODER, L. Kompendium der Veterinar Anato. mie. Jena, Gustav Fischer, 1962, v.2.

25 - SISSON, S. \& GROSSMANN, J.D. Anatomia de los animales domesticos. Barcelona, Salvat Editores, 1965.

26 - ZIMMERL, U. Anatomia topográfica veterinaria. Milano, Francesco Vallardi, 1949, v.2.

Recebido para publicação em 19-5-77 Aprovado para publicação em 3-8-77 